\title{
OPEN SCIENCE: PAOLO BUDRONI BEANTWORTET \\ 10 FRAGEN VON BRUNO BAUER ZUR BEDEUTUNG VON \\ FORSCHUNGSDATENMANAGEMENT SOWIE ZUR \\ ENTWICKLUNG DER EUROPEAN OPEN SCIENCE CLOUD
}

\author{
von Bruno Bauer und Paolo Budroni
}

Zusammenfassung: Paolo Budroni ist seit 1991 im universitären Bereich tätig. Zunächst beantwortet er Fragen über seinen beruflichen Zugang zu den Themen Forschungsdokumentation und Forschungsdatenmanagement. Angesprochen werden auch nationale und internationale Projekte und Initiativen zum Forschungsdatenmanagement, wobei der Fokus auf der Darstellung der European Open Science Cloud liegt. Zuletzt richtet Budroni einen Appell an die Bibliotheken, sich verstärkt in die Umsetzung des Konzeptes von Open Science einzubringen.

Schlagwörter: Open Science; Forschungsdokumentationssystem; Forschungsdaten; Forschungsdatenmanagement; European Open Science Cloud; EOSC; Interview

\section{OPEN SCIENCE: PAOLO BUDRONI ANSWERS TEN QUESTIONS FROM BRUNO BAUER ON THE IMPORTANCE OF RESEARCH DATA MANAGEMENT AND THE DEVELOPMENT OF THE EUROPEAN OPEN SCIENCE CLOUD}

Abstract: Paolo Budroni has been working in the university sector since 1991. At the beginning he answers questions about his professional career in the field of research documentation and research data management. The interview addresses national and international projects and initiatives for research data management with a focus on the European Open Science Cloud. Finally, Budroni encourages libraries to actively implement the concept of Open Science.

Keywords: Open Science; research information system; research data; research data management; European Open Science Cloud; EOSC; interview

DOI: https://doi.org/10.31263/voebm.v73i2.4013

(C) Bruno Bauer, Paolo Budroni

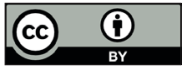

Dieses Werk ist - exkl. einzelner Logos und Abbildungen - lizenziert unter einer Creative-Commons-Lizenz Namensnennung 4.0 International 


\section{Forschungsdokumentationssystem}

Bauer: Lieber Paolo, Du hast zwischen seit 1991 an der Universität Wien in den Bereichen Forschungsdokumentation sowie Repositorium und digitale Langzeitarchivierung - Stichwort Phaidra - jeweils in einer Pionierrolle Aufbauarbeit in aus damaliger Sicht wichtigen Zukunftsfeldern betrieben. Was waren Deine Motive, Dich mit den Themen Forschungsinfrastrukturen und Forschungsdatenmanagement zu beschäftigen?

Budroni: 1991, als ich dazu kam, war die Universität Wien in einer nachhaltigen Umbruchphase. Viele der heute tätigen Organisationseinheiten und Initiativen sind bereits damals - noch vor dem neuen UG 2002 - entstanden. Rektor Karl RudolfWernhart hatte mich an die Universität geholt, um in dem von ihm geleiteten und neu gegründeten Außeninstitut zu arbeiten, und ich wurde gleich mit dem jungen, neugewählten Rektor, Alfred Ebenbauer, 48 Jahre alt, voller Tatendrang, bekannt gemacht. Er sollte mein direkter Vorgesetzter für die darauffolgenden sechs Jahre werden. Damals war das Rektorat der Universität Wien unendlich viel kleiner und überschaubarer als heute, wir waren nur vier, dann später fünf Mitarbeiter, so waren die Aufgabenbereiche stark bereichsübergreifend und nicht so vertikal wie heute aufgestellt. Außerdem verfügte der Rektor über kein eigenes Budget.

Unter diesen Voraussetzungen ergaben sich für mich drei Aufträge, die folgendermaßen kommuniziert wurden: „Die Universität Wien weiß derzeit nicht, wer an welchen Themen an den Instituten forscht: bitte bauen Sie eine dementsprechende Datenbasis auf. Auch habe ich als Rektor kein Budget dafür: bitte sorgen Sie dafür, dass wir verstärkt Drittmittelakquisition an der Uni einführen. Die Uni hat auch kein Medium zur Verfügung, über das Forschungsmarketing betrieben und der Output der Forschungsergebnisse bekannt gemacht werden kann. Für den Aufbau dieses Mediums werden wir einen Teil der Erlöse aus dem Fundraising einsetzen, und ich beauftrage Sie hiermit, neue Wege zu beschreiten."

Einige Produkte der daraus resultierenden Tätigkeit, die sich in den darauffolgenden vier Jahren voll entfaltetet hat, waren die Gründung des Alumniverbandes (um beispielsweise „people-raising“ zu initiieren), die Gründung des Mediums „Die Universität/The University/La Universidad“, welches ab dem Jahr 1995 auch dreisprachig als Printausgabe und als Online-Medium erschien, und zu 100 Prozent aus Fundraisingtätigkeit finanziert wurde. Ich war einer der zwei Herausgeber. Die Printausgabe der Zeitschrift hatte als Sponsoren die Bank Austria, AGIP und den Kurier. Bemerkenswerterweise ist die Universität ein paar Monate früher als den Kurier online gegangen. Grundlage der genannten Tätigkeiten war das Aufspüren der Forschungsaktivitäten an der Universität Wien, was systematisch erfolgte. 
Ich erwähne diese Umstände, weil sie miteinander verknüpft sind. Wir konnten im dreisprachigen Medium den Output der Universität Wien in drei Sprachen gut darstellen, und durch Werbemaßnahmen jene Drittmittel lukrieren, die für den weiteren Ausbau der Forschungsdatenbank notwendig waren. Durch den Alumniverband konnten wir überdies wichtige Netzwerke aufbauen.

Nun zur ersten Forschungsdatenbank der Universität Wien, dem ersten CRIS (Current Research Information System). Es gab zu jenem Zeitpunkt mit FODOK (Forschungsdokumentation) bereits eine Initiative der TU Wien, mit der beide Rektoren Wernhart und Ebenbauer, nicht glücklich waren, denn die Uni Wien lieferte Daten, hatte jedoch keinen direkten Zugang dazu.

Die Konzeption des neuen Systems beruhte auf folgenden Überlegungen: 1) das neue System musste dezentral nach neuesten Erkenntnissen aufgebaut werden, 2) die rechtliche Sicherheit über die Daten musste gewährleitet werden, dabei musste die Universität Wien die Hoheit über die Daten erhalten, und 3) das System musste immer folgenden fünf Anforderungen entsprechen: Leichtigkeit, Schnelligkeit, Genauigkeit, Anschaulichkeit, Vielschichtigkeit. Im Jahr 1997 habe ich im Rahmen eines postgradualen Studiums eine Abschlussarbeit verfasst, die diese Prinzipien beim Aufbau des Systems erläutern. ${ }^{1}$

Für das neue CRIS kamen einige Instrumente zur Anwendung, die zum damaligen Zeitpunkt für diese Form des Arbeitens bahnbrechend waren: Wir führten ein Pilotprojekt an der SOWI Fakultät durch, wo auch die Informatik beheimatet war. Wir verwendeten CERIF (Common European Research Information Format), empfohlen von der Kommission vom 6. Mai 1991 zur Harmonisierung der Datenbanken auf dem Gebiet der Forschung und technologischen Entwicklung in der Gemeinschaft - damals war Österreich noch nicht Mitglied der Europäischen Gemeinschaft - und das „Frascati Manual“, publiziert von der OECD (Organisation for Economic Cooperation and Development). Wir setzten es durch, dass die wichtigsten Einträge zu den datasets auch auf Englisch geliefert wurden, was damals einer kleinen Revolution entsprach, und es wurden Thesauren eingeführt, darunter der mehrsprachige und multidisziplinäre Thesaurus EUROVOC ${ }^{2}$, die Klassifikation OESTAT, die damals schon beim FWF gebräuchlich war, und als letztes sei noch die ISO Norm 2709 erwähnt. Auch wurden insgesamt über 20 Personen angeheuert, die beim Aufbau der Datenbasis systemisch arbeiteten - z.B. durch persönliche Befragungen. Die Arbeiten an der SOWI Fakultät wurden rasch beendet und wir gingen dann über, die Medizinische Fakultät - heute Medizinische Universität Wien - zu bearbeiten. Das implizierte in allen Fällen eine enge Zusammenarbeit mit den jeweiligen Fakultätsleitungen - so auch mit dem späteren Rektor Wolfgang Schütz. 
Die Arbeit war in diesem Umfang nur möglich, weil wir am Außeninstitut bereits damals an integrierte Wissensverarbeitung und Know-how-Transfer dachten. Natürlich waren wir eingebettet im universitären Geschehen und profitierten auch von den unterschiedlichen Rückmeldungen seitens jener, mit denen wir - zur Bewältigung der Arbeit - in Kontakt traten. Einige seien hier noch genannt: Prof. A-Min Tjoa, Informatik Uni Wien, den ich heute noch an der TU Wien beruflich im Rahmen des EOSC Secretariat am IFS (Information and Software Engineering) treffe. Prof. Tjoa wurde später auch mein wissenschaftlicher Betreuer beim Verfassen der Abschlussarbeit an der Europaakademie des Bundes. Seinem Ratschlag folgend beschäftigte ich mich mit dem Thema „reengineering the corporation“, und einer seiner Studenten programmierte die Forschungsdatenbank der Uni Wien. Das ZID der Universität Wien hingegen barg ein immenses Kapital an Wissen, was Infrastrukturen betraf, und hier sei ein Name erwähnt, der später bei der sogenannten „Phaidra-Experience“ eine Rolle spielen sollte: Peter Marksteiner. Aus der Bibliothek sei noch der damalige Vizedirektor, Paul Rauchbauer erwähnt. Sie alle unterstützten mit ihrem Wissen den Aufbau der Datenbasis.

Wir waren erfolgreich. Die Österreichische Akademie der Wissenschaften meldete sich bei uns und fragte an, ob wir sämtliche Stellen der ÖAW österreichweit besuchen konnten, um auch bei ihnen, das System der Uni Wien einzusetzen und den Forschungsoutput der ÖAW zu erfassen. Später kam auch die VetMed dazu.

Die vielleicht wichtigsten Erkenntnisse jener Jahre sind durch einige Anforderungen an die Forschungsdaten wiederzugeben:

Sie sollen interoperabel und leicht auffindbar sein. Dass dies auch damals möglich war, hat die Nachnutzung der Daten bei der Zeitung der Universität Wien deutlich gezeigt.

Alle erfassten Daten sollen rechtlich abgesichert sein, vor allem was ihre Nachnutzungsmöglichkeiten betrifft.

Die Infrastrukturen, in denen die Daten untergebracht werden sollen stabil und resilient sein. Wir sehen heute in der COVID19-Krise, wie wichtig resiliente Infrastrukturen sind.

\section{Forschungsdaten}

Bauer: Zum Thema „Forschungsdaten" haben viele, die sich mit dem Thema nur peripher beschäftigen, unterschiedliche Vorstellungen. Wie würdest Du den Begriff „Forschungsdaten" erklären? 
Budroni: Bezüglich der Definition von Forschungsdaten: da möchte ich verweisen auf die Definitionen, die wir im H2020 Projekt LEARN ${ }^{3}$ verwendet haben. Dabei haben wir ein „Toolkit of Best Practice for Research Data Management" verfasst und publiziert, bei dem wir eine ausgezeichnete Definition von Forschungsdaten angeben:

Three further approaches, each dealing with different aspects of research data, may help to find the proper definition for individual research institutions:

a. According to the LERU Roadmap for Research Data4 (LERU Research Data Working Group, Advice Paper No. 14 - December 2013):

"Research data, from the point of view of the institution with a responsibility for managing the data, includes: All data which is created by researchers in the course of their work, and for which the institution has a curational responsibility for at least as long as the code and relevant archives/record keeping acts require, and third-party data which have originated within the institution or come from elsewhere."

b. The Australian Griffith University presents the following definition:

"Research data are factual records, which may take the form of numbers, symbols, text, images or sounds, which are used as primary sources for research, which are commonly accepted in the research community as necessary to validate research findings."

c. The University of Minnesota definition of research data:

"Research data are data in any format or medium that relate to or support research, scholarship, or artistic activity. They can be classified as:

- Raw or primary data: information recorded as notes, images, video footage, paper surveys, computer files, etc.

- Processed data: analyses, descriptions, and conclusions prepared as reports or paper

- Published data: information distributed to people beyond those involved in data acquisition and administration." 4

Zum Themenkomplex Forschungsdaten möchte ich noch folgendes festhalten:

- Das Thema Research Data ist immer mit dem Thema Educational Resources zu verbinden und zu behandeln, weiters sind Publikationen als Metadaten zu den Daten zu betrachten. Diese Aussage mag provokativ klingen, ist aber ernst gemeint. Denn im Laufe ihres Life Cycle erfahren die Daten Veränderungen in ihrem Zustand. Sie können den Grad ihrer „accessibility“ verändern und somit können sie immer wieder neu angereichert, neu aggregiert, und neu interpretiert werden, z.B. je nachdem ob ein Mensch oder eine Maschine sie analysiert 
und interpretiert. Dies führ zu neuen Metadaten, somit auch zu neuen Publikationen.

- Das, was wir heute als Forschungsdaten bezeichnen, sollte zumindest in folgende Kategorien unterteilt werden: a) Content; b) dazugehörige Metadaten; c) Software (Applikationen sind auch Daten, und sie bedienen die Logiken nach denen Daten gemanagt oder interpretiert, oder zugänglich gemacht werden; d) Persistent Identifiers sollten als eigene Digitale Objekte erfasst werden können. Und als letzte Kategorie e) weise ich auf die Querverbindungen zwischen den Ressourcen hin (die sogenannten Links): derzeit zeigt es sich, dass es vielleicht die Links sind, die den wertvollsten Bestand darstellen.

- Das hätte zur Folge, dass in den derzeitigen Repositorien 1) die Metadaten über eigene Persistent Identifiers verfügen würden, denn Metadaten können eigene intellektuelle Entitäten darstellen, und 2) Links als digitale Objekte definiert werden sollten, und somit metadatisiert und mit persistenten Indikatoren versehen werden müssten, somit wären alle links zu einem Thema durch eine Suchaktion auffindbar.

- Zu guter Letzt stelle ich folgende Forderung auf: die sogenannte „machine actionability" der Daten sollte auf ein Maximum forciert werden, COVID19 zeigt auf, wie relevant diese „machine actionability“ ist.

\section{E-Infrastructures Austria}

Bauer: Mit der Finanzierung des Hochschulraumstrukturmittelprojektes e-Infrastructures Austria (Laufzeit: 2014-2016) durch das österreichische Wissenschaftsministerium wurde es möglich, das Thema „Forschungsdatenmanagement" an den Hochschulen und Forschungseinrichtungen in Österreich zu verankern. Worin siehst Du - aus der Perspektive des Leiters dieses Projektes - den wesentlichen Nutzen von e-Infrastructures Austria für die weitere Entwicklung von Forschungsdatenmanagement in Österreich?

Budroni: Im heurigen Jahr wurden wir vom BMBWF aufgefordert, das damalige Projekt e-Infrastructures Austria Phase I zu bewerten. Ich glaube, dass aus heutiger Sicht einige große Folgewirkungen beobachtet werden können:

1. Ganz oben wäre zu setzen - und das kannst Du mir als damaliger und sehr erfolgreicher Vorsitzender der Generalversammlung der Mitglieder im Projekt bestätigen: Das Projekt e-Infrastructures 
Austria hat ein funktionierendes Netzwerk an Kompetenzen hervorgebracht, das meiner Ansicht nach einmalig in Europa ist. Einundzwanzig österreichische Universitäten und fünf weitere Partner arbeiteten gemeinsam an ausgewählten Themen. Das war eine großartige Erfahrung. Im Laufe meiner darauffolgenden internationalen Tätigkeit wurde ich auf dieses besondere Netzwerk mehrfach angesprochen. Die Kollegen von SwissUniversities haben uns besucht, die Kollegen aus dem italienischen Gesundheitsministerium haben die Forschungsdatenerhebung für 62 italienische Einrichtungen - genauso wie in Österreich - durchgeführt. Nebenbei angemerkt: in der Studie ist auch ein Vergleich zwischen den Ergebnissen der österr. Forschungsdatenumfrage und der italienischen gewidmet. ${ }^{5}$ Wir sind eingeladen worden das Projekt e-Infrastructures Austria in vielen Ländern vorzustellen, auch nach seinem Ende - in den Niederlanden, in Dänemark, in Norwegen, sogar bei einer UN Conference in der Karibik (remote).

2. Die zweite Folgewirkung ist, dass drei wesentliche Themenbereiche nun als normal und notwendig erachtet werden. Diese sind: Forschungsdatenmanagement. Wir alle sind uns der Bedeutung des Forschungsdatenmanagements bewusst, zwei österreichische Unis verfügen bereits über erfolgreiche Forschungsdatenzentren. Ein weiteres Thema ist die Einführung von Policies zum Forschungsdatenmanagement. Ihr an der MedUniWien seid auch unter den ersten vier österreichischen Universitäten gewesen, die eine Policy eingeführt haben. Der darauffolgende logische Schritt war das nächste HRSM-Projekt, zur Etablierung von Kompetenzen im Bereich Educational Resources: So entstand das Projekt Open Education Austria. Diese Folgewirkung ist sehr bedeutend: im Rahmen der RDA Generalversammlung 2018 in Botswana herrschte ein reges Interesse um die Educational Resources, und Open Education Austria ist im Mittelpunkt dieses Interesse gestanden. Ich möchte noch die Aufmerksamkeit auf einen weiteren Umstand lenken, und ich glaube das wichtigste Ergebnis ist: e-Infrastructures Austria hat klar und deutlich gezeigt, dass die Community der österreichischen wissenschaftlichen Bibliotheken über ein sehr hohes Niveau an Kompetenzen verfügt, um so komplexe Projekte erfolgreich national und international jahrelang durchführen zu können. Sämtliche Impulse zum Forschungsdatenmanagement sind in Österreich aus den Bibliotheken hervorgegangen, so auch die zwei bereits erwähnten Forschungsdatenzentren - an der TU Wien und der TU Graz. 


\section{Nationale und internationale Projekte und Initiativen zum Forschungs- dastenmanagement}

Bauer: Aufgrund Deiner Pionierarbeit bei E-Infrastructures Austria hast Du Dir eine Expertise für den Bereich des Forschungsdatenmanagements erarbeitet, das Dich zu einem gefragten Experten für verschiedene nationale und internationale Projekte und Initiativen zum Thema Forschungsdatenmanagement gemacht hat - ich nenne hier nur LEARN und NADRE sowie Deine Funktionen bei RDA Austria und E-IRG. Welche Ziele wurden bzw. werden mit diesen - und gegebenenfalls anderen von Dir ebenfalls betriebenen - Projekten und Initiativen zum Forschungsdatenmanagement, verfolgt?

Budroni: Die wichtigsten Ziele sind: erstens die Vernetzung der österreichischen RIs (Research Infrastructures) noch stärker zu promoten, zweitens soll dies nach Möglichkeit von den Bibliotheken aus geschehen und drittens als vielleicht wichtigster Punkt soll die Kultur der Zusammenarbeit beim Forschungsdatenmanagement gefördert werden. Das kann mit diesem Begriff zusammengefasst werden: FAIRisierung der Daten und der Services. Hier ein paar Beispiele von Projekten/Initiativen, die ich entweder im Auftrag der UB Wien geleitet, akquiriert, oder bei deren Akquise ich wesentlich beteiligt war und bei denen diese drei Leitgedanken wirkungsvoll eingesetzt wurden.

Zuerst einmal Phaidra. Das war eine mehr als zehnjährige Erfahrung, die gemeinsam mit Thomas Wana, ZID Uni Wien, beim Schreiben des Konzeptes, der Beschreibung der Architektur, dem Aufsetzen des Pflichtenheftes begann und im Laufe der Jahre zur Bildung eines Netzwerkes an digitalen Infrastrukturen, zu Schulungen und Workshops und zum Kennenlernen von unterschiedlichen Communities - alles unter dem Namen Phaidra - führte. Diese Infrastrukturen befinden sich heute in fünf Ländern und betreffen über 16 Repositorien.

Neben den bereits erwähnten e-Infrastructures Austria, Open Education Austria und LEARN sind im Sinne von Open Science ebenfalls erwähnenswert: Das Projekt OpenAIRE, das heute aus der europäischen Landschaft nicht mehr wegzudenken ist, das Projekt Europeana Libraries - dabei geht es um Daten rund um das Weltkulturerbe, das Tempus Project in Western Balkan Countries, das den Wiederaufbau einer Infrastruktur zum Forschungsdatenmanagement in Serbien, Montenegro, Bosnien zum Ziel hat, das H2020-Projekt EOSC Secretariat zur Unterstützung des EOSC Executive Boards bei der Einführung der EOSC in Europa, das Projekt EOSC Pillar zur Unterstützung des EOSC building process in Österreich, Belgien, Frankreich, Deutschland und Italien und die zwei neuesten H2020-Projekte FAIR-Move und CO-Versatile. FAIR-Move ist 
von der Kommission derzeit in den Status on-hold gesetzt worden, dabei geht es um das europaweite Management von Open Data aus dem Bereich Klima-Daten. CO-Versatile ist bereits bewilligt, und entsteht im Rahmen einer europaweiten industriellen Kooperation. Dabei handelt es sich um eine COVID19-Urgent Action der Europäischen Kommission, bei der der europaweite Einsatz von Daten derart optimiert werden soll, dass die Produktion von z.B. Schutzmasken und Schutzanzügen, wie auch sogenannte PPEs (Personal Protection Equipments) in nächster Zukunft reibungsloser und rascher erfolgen kann. Auch diese zwei letztgenannten Projekte sind wieder einmal von einer Universitätsbibliothek aus akquiriert worden, und werden in Zukunft von dort aus gemanagt, in diesem Fall ist es die TU Wien Bibliothek.

Eine wesentlich anders gelagerte Angelegenheit ist das von Dir erwähnte NADRE ${ }^{6}$ (National Academic Digital Repository of Ethiopia). Diese Initiative ist komplex und in eine Reihe von weiteren internationalen Aktivitäten eingebettet. In Äthiopien haben wir gemeinsam mit der Universität Catania/Istituto Nazionale di Fisica Nucleare, unterstützt von der italienischen Rektorenkonferenz, Trainings zum Thema Data Stewardship und FAIR angeboten und durchgeführt. Finanziert wurde das Projekt von der deutschen Gesellschaft für Internationale Zusammenarbeit. Dort haben wir auch gemeinsam am Aufbau der digitalen Infrastruktur und beim Launch mitgewirkt. Zielgruppe waren die 46 äthiopischen Universitäten. Der darüber liegende Kontext ist die europäische Agentur TAIEX ${ }^{7}$, in dessen Auftrag ich in der Türkei und im Libanon war, beide Male um bei mehrtägigen Workshops für Regierungsvertreter Knowhow-Transfer zu betreiben. Dabei ging es um das Thema Open Data und die Implementierung der PSI Richtlinie in diesem außereuropäischen Kontext.

Durch all diese Aktivitäten zieht sich ein roter Faden, symbolisch durch die „wissenschaftliche Bibliothek" - Research Library - dargestellt.

Das Thema Bibliothek ist insofern faszinierend, weil es manchmal vorkommt, dass Universitätsleitungen die neuen Rollen und Aufgabenprofile der Bibliotheken entweder nicht zur Gänze wahrnehmen bzw. nicht immer korrekt einschätzen. Ich sehe es daher als Ziel meiner Tätigkeit, innovative Prozesse erstens zu initiieren und weiters auf die relevante Rolle der Universitätsbibliotheken aufmerksam zu machen bzw. diese zu stärken. Research Data Alliance Austria National Node ist genauso eine Erfolgstory wie die österreichische Teilnahme an der e-Infrastructures Reflection Group. ${ }^{8}$ Zum ersten Mal in seiner Geschichte wird dieses wichtige europäische Advisory Board, das von den Member States and Associated Countries bestellt wird, nicht von einem Vertreter einer technischen Infrastruktur geleitet, sondern von einem Vertreter einer österreichischen Bibliothek. ${ }^{9}$ 
Und es war - mit der Universitätsbibliothek der Universität Wien - auch an einer österreichischen Bibliothek, an der im November 2018 die European Open Science Cloud gelauncht wurde.

\section{European Open Science Cloud (EOSC)}

Bauer: Aufgrund des Begriffs European Open Science Cloud könnte man versucht sein zu denken, es handelt sich bei der EOSC um ein auf Europa begrenztes Projekt, um eine "Cloud", die in Brüssel entwickelt wird, um ein Konzept, das ausschließlich Daten und Ergebnisse von Forschungen enthält, die „open“ sind. Was ist aus Deiner Sicht die korrekte Definition der EOSC?

Budroni: Ja, richtig, das war ein denkwürdiges Erlebnis, als im November 2018 Minister Heinz Fassmann, begleitet von der EU-Kommission und von den Vertretern der bulgarischen und rumänischen EU-Präsidentschaft im großen Lesesaal der Universitätsbibliothek Wien die „Vienna Declaration on the European Open Science Cloud" deklamierte, worauf diese anschließend auch angenommen wurde. ${ }^{10}$

Die Antwort auf Deine Frage ist gleichzeitig einfach und doch komplex, wenn es um die Details geht.

Zuerst einmal die rasche und konzise Antwort. Gemeinsam mit Jean-Claude Burgelman („Vater" der EOSC) und mit Michel Schouppe (Open Science Unit der Kommission und „Patenonkel“ der EOSC) haben wir einen Artikel verfasst, der im Jahr 2019 der am meisten heruntergeladene Beitrag bei der Zeitschrift ABI Technik war. ${ }^{11}$ Dieser Beitrag beschreibt die EOSC wie folgt: „Die EOSC ist kein Projekt, und keine Cloud, sondern ein Prozess. Dabei geht es um eine europaweite Föderation von bereits existierenden Infrastrukturen und Services, eingebettet in einem Web von FAIR-Services und Daten".

Eine weitere Definition wurde in einem im Jänner 2020 erschienen Artikel geboten, in dem auch das österreichische Engagement im EOSC building process genauer dargestellt wird. ${ }^{12}$

Woraus resultiert also die Komplexität der EOSC? Sie ergibt sich aus der Heterogenität Europas, seiner 27+1 handelnden Akteure, der EU-Kommission sowie der zehn Associated Countries, die auch mit dabei sind, z.B. Schweiz, Norwegen, Israel, Türkei. Und weiter durch das Zusammenspiel der folgenden sechs Ebenen bzw. lines of actions: die juristische Ebene („Definition of Rules of Participation“ für alle in Europa), die nachhaltige Entwicklung der EOSC (Sustainable EOSC), die Analyse der vorhandenen Infrastructures and Policies (Landscape analysis), der Aufbau von Kompe- 
tenzen (Skills development), die Architektur der Infrastrukturen (Architecture) sowie die FAIRisierung von Daten und Services (FAIR).

Die sechs genannten Ebenen entsprechen auch den sechs Working Groups des EOSC Executive Boards. Selbst bin ich in zwei dieser Gruppen tätig: in der Landscape Analysis ${ }^{13}$ und in der Sustainability Working Group $^{14}$. Weiters koordiniere ich eine kleine Task Force, deren Aufgabe es ist, die Logik für das Generieren von „Key Performance Indicators (KPIs) for Infrastructures" zu definieren.

Jede der Working Groups besteht aus zirka 30 bis 40 Mitglieder. Dazu kommen zirka fünfzig H2020 EOSC building projects. Wir alle gemeinsam sollen im Laufe von 24 Monaten die EOSC operativ werden lassen. So gesehen ist die EOSC tatsächlich ein europäisches Konstrukt, Ausdruck des gemeinsamen Handelns.

Was kann man sich unter EOSC building projects vorstellen?

Hier ein paar Namen, die geläufig sein dürften: EOSC Pillar, EOSC Nordic, EOSC Synergy, FAIRsFAIR, OpenAIRE, RDA Europe, „ESFRI Cluster-Projects", EOSC Hub, FREYA ... Sie alle haben eins gemeinsam: ihre Mitglieder und Partner arbeiten gleichzeitig, wie alle anderen auch, am Aufbau der EOSC. Unterstützend wirkt das H2020 Projekt EOSC Secretariat - dieses ist in Österreich an der TU Wien angesiedelt. Ich bin dort im Bereich „Researchers Engagement" tätig, konkreter beschäftige ich mich mit den universitären Netzwerken EUA, The Guild, LERU, The Coimbra Group, CESAER, SwissUniversities und mit Science Europe.

Das EOSC Secretariat unterstützt das EOSC Executive Board ${ }^{15}$, das wiederum regelmäßig, alle paar Wochen das EOSC Governance Board ${ }^{16}$ über die laufenden Aktivitäten informiert. Der österreichische Vertreter im Governance Board ist Stefan Hanslik vom BMBWF. In all diesen Zusammenhängen ist es extrem wichtig, dass eine regelmäßige Rückkoppelung auf nationaler Ebene zwischen den interessierten Stakeholdern stattfindet. Deshalb hat man in Österreich das sogenannten Austrian EOSC Café formiert, welche sich auf rein informeller Basis regelmäßig trifft.

So findet ein Austausch über europäische und nationale Aktivitäten statt, bei dem ein gemeinsamer Wissensstand entsteht. Mit dabei sind einige jener Vertreterlnnen, die regelmäßig an den europäischen Treffen teilnehmen, wie z.B. Delegierte von vier Ministerien, der Forschungsförderer FWF und FFG, des EOSC Secretariats, von EOSC Pillar, von ACOnet und E-IRG sowie der TU Wien und RDA Austria. Durch diesen Austausch sind z.B. die Country Profiles entstanden, die in der WG Landscape verwendet werden, aber auch der offizielle österreichische Input zu den Statuten der EOSC Association, die vor wenigen Tagen nach belgischem Recht gegründet wurde. ${ }^{17}$ 


\section{Inhaltliches Konzept der EOSC}

Bauer: Du hast nun ausgeführt, welche Ziele die EOSC verfolgt. Offen blieben bisher aber noch die wichtigen Fragen nach der Konzeption von Daten und Diensten in der EOSC sowie der dahinterliegenden Infrastruktur. Wer ist für die Schaffung und Weiterentwicklung der Infrastruktur verantwortlich? Wer entscheidet, was ,in die EOSC hineinkommt"? Wie wird die für die Forschung essentielle Qualitätssicherung der Inhalte der EOSC sichergestellt?

Budroni: Die hier gestellten Fragen sind leicht aus der Definition der EOSC zu beantworten: die Föderation bestehender Infrastrukturen und Services steht im Mittelpunkt der EOSC, und diese Infrastrukturen und Services werden in den Staaten definiert, die Mitglieder der EOSC Association sind. Das bedeutet, dass jeder entsprechend dem eigenen Bedarf Infrastrukturen und Services bereitstellt, die anschließend föderiert werden. Für die Schaffung und Weiterentwicklung sind die jeweiligen Betreiber zuständig. Die bereits genannten $R u$ les of Participation legen Eintritts- und Austrittsregeln fest. Dabei ist zu beachten, dass die EOSC für alle da sein soll: Forscher-Communities, Infrastrukturbetreiber, Servicebetreiber, öffentliche Einrichtungen, Industrie, N.G.O.s, ja sogar einzelne Bürger (Citizen Scientists). Die Qualitätssicherung soll über die Anwendung von sogenannten Critical Success Factors erfolgen. Ihnen werden auch die dafür notwendigen KPIs zugeordnet. Die für die Forschung essentielle Qualitätssicherung wird über unterschiedliche Instrumente erfolgen. Die Working Group Landscape führt gerade ihre Arbeiten zu Ende, bei denen sogenannte Country Profiles ermittelt und vom Digital Curation Centre (UK) analysiert werden. Die Föderation der nationalen Research Infrastructures wird auch untersucht, dies erfolgt gemeinsam in der Working Group Sustainability. Derzeit entstehen auch Empfehlungen zum Thema Better Research. Diese Initiativen haben es sich zum Ziel gemacht nicht nur den Status Quo zu ermitteln, sondern auch Empfehlungen auszuarbeiten, die qualitätssichernde Maßnahmen mit sich bringen. Die Qualität der Forschung hängt auch von Themen wie der Einführung von Policies, z.B. einer nationalen Policy zu EOSC-bildenden Maßnahmen oder eine Policy zu Open Science oder eine Policy zu Educational Resources ab. Wichtig ist auch, ob Forschungsförderer qualitätssichernde Instrumente einsetzen bzw. einfordern. Zuletzt hängt die Qualität der Forschung auch von der Qualität der Services und der Infrastrukturen $\mathrm{ab}$, wie auch von der Beachtung des juristischen Rahmens.

Hier einen kurzen Auszug aus dem Instrumentarium, zur Einführung von Qualitätsfördernden Maßnahmen, aus dem Bereich Sustainability entnommen: 
- A shared open science policy framework

- Authentication and Authorization Interoperability (AAI) framework

- Data access framework

- Service management and access framework

- A minimum legal metadata framework, for ensuring openness and interoperability, privacy and security

- An open metrics framework

- PID Services

- Help-desk (e.g. consultancy on EOSC-Core services)

- Portal: an EOSC Service implementing a web portal providing one form of accessing and using the EOSC Resources.

Vorhin habe ich die Statuten der EOSC Association erwähnt. Diese Statuten sind klar in ihren Aussagen. Neben den ordentlichen Mitgliedern gibt es auch die sogenannten Observers. Die Frist, um sich als Mitglied oder Observer anzumelden, ist auf den 15. Oktober 2020 erstreckt worden. Man erwartet viele Interessenskundgebungen. Die sogenannte pre-General Assembly ist für den 28. Oktober 2020 vorgesehen, die erste General Assembly wird am 17. Dezember 2020 stattfinden. Die Teilnahme an der EOSC wird in den oben angeführten Rules of Participation geregelt. Grob dargestellt werden in den kommenden Wochen, drei wesentliche Dinge geschehen: man wird an den Bylaws (Statuten) weiterarbeiten, um die Selbstregulierungsmechanismen optimieren zu können, man wird ein MoU (Memorandum of Understanding) definieren, was irgendwie an eine Konsortialvereinbarung erinnern soll, und man wird bis Ende Oktober an der Fertigstellung der SRIA (Strategic Research and Innovation Agenda) arbeiten. Die SRIA stellt ein wesentliches Element bei der Bildung der EOSC dar. Denn sie enthält die Ergebnisse der Arbeit in den sechs Working Groups und legt in unterschiedlichen Kapiteln die Rollen, die Verantwortungsbereiche und die Zeitpläne für die nächsten Jahre fest.

Bis Mitte September fand eine Public Consultation über die wesentlichen Teile der SRIA zur EOSC statt, dementsprechende Aufrufe waren europaweit ausgegangen. Die österreichischen Universitäten wurden beispielsweise über die europäischen Universitätsnetzwerke informiert, so auch - über die EUA - die uniko.

\section{Finanzierung und Gremien der EOSC}

Bauer: Wenn ich mit Kolleginnen und Kollegen über die EOSC spreche, so ist mein Eindruck, dass bei vielen die Erwartungshaltung mitschwingt: für die Finanzierung 
der EOSC sorgt Brüssel. In der Realität sieht sich die Europäische Kommission allerdings durchaus nicht als Dauersponsor der EOSC. In welcher Größenordnung wurde die EOSC bisher von der Europäischen Kommission finanziell unterstützt und wie lange laufen die Förderungen? Welche konkreten Überlegungen gibt es derzeit, um eine nachhaltige Finanzierung der EOSC sicherzustellen? Und spätestens beim Thema Finanzierung stellt sich auch die Frage nach den Entscheidungsgremien für das betreffende Projekt. Welche Gremien sind bei der EOSC für die inhaltliche Ausrichtung sowie für die erforderlichen administrativen Aufgaben zuständig? Wie erfolgt deren Zusammensetzung?

Budroni: Diese Erwartungshaltung kann mitunter begründet sein, denn bei der EOSC verhält es sich derzeit sehr ähnlich wie beim Thema Open Data. Für die Endverbraucher sind die Daten offen und frei zugänglich. Die Kosten für deren Entstehung und Wartung wurden beispielsweise vorher von anderen getragen. Ob dabei Brüssel mitfinanziert oder nicht hängt ab von dem, was man gerade konsumiert oder verwendet. Damit meine ich Dienste, Infrastrukturen oder Daten. Das bedeutet, dass die Quellen der Finanzierung unterschiedliche sein werden, die Endverbraucher - vor allem in den Forscher-Communities - sollten so wenig wie möglich bzw. keine Kosten tragen. Das Stichwort dazu ist: „be free at the point of use“, das bedeutet jedoch nicht, dass sie kostenlos aufgebaut wurden, oder kostenlos gewartet oder zur Verfügung gestellt werden. Ein beliebtes Beispiel sind die derzeitigen Regelungen für Eduroam: jeder, der über einen Zugang verfügt, bezahlt nicht bzw. hat den Eindruck nicht zahlen zu müssen. Eduroam ist an den Unis generell vorhanden, jederzeit einwählbar, sicher, und „trusted“. Man muss auch nicht lange überlegen, um verstehen zu können, wie es funktioniert. So ähnlich sollte man sich in der Regel den Zugang und die Nutzung der EOSC vorstellen, vorausgesetzt, man gehöre zur wirklich großen Community der User. Tatsächlich hat die Europäische Kommission bis jetzt sehr viel Geld investiert, in der Größenordnung von mehreren hundert Millionen Euro. Weitere Finanzierungsschübe werden folgen. Allein die EOSC building projects der unterschiedlichen INFRAEOSC Projects haben mehr als 50 EOSC Building Initiativen mit einer Projektdauer von bis zu drei Jahren mit sich gebracht. Die meisten dieser Projekte gehen in zirka zehn Monaten zu Ende. Mit der nachhaltigen Entwicklung der EOSC befasst sich derzeit am intensivsten die Working Group Sustainability. Spätestens Ende Oktober 2020, also in wenigen Tagen werden diese geplanten Maßnahmen „publicly available“ sein.

In diesem Zusammenhang spricht man von der MVE (Minimum Viable EOSC), wobei in der ersten Phase die Föderation stattfinden soll. Dieses Konstrukt sieht annähernd wie eine Zwiebel aus, bestehend aus einem 
Kern (EOSC Core) und einer Schale (EOSC-Exchange). Dazwischen sind die sogenannten Federated Data vorhanden. EOSC Core sorgt für die minimal notwendigen Funktionalitäten, die für Open Science notwendig sind, fachübergreifend und staatenübergreifend. EOSC-Core wird für die Bereiche "discovery, share, access and re-use" sein. An diesem Core sollen jene Dienste andocken, die für den Transfer von Daten, das Prozessieren von Daten, usw. zuständig sein werden. Diese Dienste, die auch konkurrierend sein können, werden von EOSC-Exchange aus operieren. Die technischen Voraussetzungen, um an EOSC-Exchange teilnehmen zu können werden die gleichen für alle sein. Die Unterschiede werden sich aus verschiedenen Policies oder juristischen Voraussetzungen ergeben, die aus den frei zugänglichen Services oder zu bezahlenden Services resultieren.

Mit Ende des Jahres erfolgt eine Übergabe der Geschäfte an die bereits erwähnte EOSC Association und die Auflösung des derzeit bestehenden EOSC Governance Board und EOSC Executive Board. Das EOSC Sekretariat wird noch weitere sechs Monate unterstützend mitwirken, danach wird auch dieses Projekt zu Ende gehen.

\section{Wissenschaftlerinnen \& Wissenschaftler und die EOSC}

Bauer: In der Selbstdarstellung der EOSC ist von 1,7 Millionen europäischen Wissenschaftlerinnen und Wissenschaftlern die Rede, denen die EOSC eine optimale Forschungsinfrastruktur bieten soll. Spricht man allerdings Wissenschaftlerinnen und Wissenschaftler auf das Thema EOSC bzw. deren Nutzen für die eigene Forschung an, so fällt die Resonanz eher ernüchternd aus - sofern sie überhaupt eine Vorstellung davon haben. Wurde bei den Verantwortlichen der EOSC nicht zu lange eine Vision bzw. ein Konzept entwickelt, ohne die Betroffenen - die Wissenschaftlerinnen und Wissenschaftler - miteinzubeziehen? Welche konkreten Überlegungen bestehen in den EOSC Gremien, um die Forschenden stärker ins EOSC-Boot zu holen?

Budroni: Der Anschein dieser Darstellung ist vielleicht etwas trügerisch. Es sind Bemühungen im Gange, möglichst viele Wissenschaftler anzusprechen. Laufend finden zahlreiche Webinars und Workshops statt. Dies erfolgt z.B. über das EOSC Sekretariat - ich empfehle, sich dort zu registrieren - und über die Universitätsnetzwerke, die Forschungsförderer und die Ministerien. Man darf dabei nicht vergessen, dass der Aufbau der EOSC sehr schnell voranschreitet, alles geschieht gleichzeitig und europaweit im Laufe von ca. zwei Jahren. So gesehen ist die Beteiligung sehr groß, umso größer wird sie sein, nach Beendigung dieser ersten Phase. 


\section{Vision von der EOSC 2030}

Bauer: Im Dezember 2020, während der deutschen Ratspräsidentschaft, wird die Implementierungsphase der EOSC abgeschlossen und die operative Phase beginnen. Nehmen wir dieses Datum als Ausgangspunkt und werfen den Blick in die vielfach strapazierte Glaskugel: Wird im Jahr 2030 die European Open Science Cloud für dann wahrscheinlich schon deutlich mehr als 1,7 Millionen europäischen Wissenschaftlerinnen und Wissenschaftlern die heute - 2020 - skizzierte optimale Arbeitsumgebung für ihre Forschung bieten? Wird dieses Konzept dann selbstbestimmt von den Universitäten und Forschungseinrichtungen getragen werden, oder aber werden kommerzielle Firmen - Stichwort: Verlage - Services und Infrastruktur bereitstellen, die von den Nutzerinnen und Nutzern zu zahlen sein werden?

Budroni: Wie bereits vorhin dargestellt, es wird unterschiedliche Methoden und Wege geben, um an EOSC teilnehmen zu können. Die europäische Gesetzgebung wird für den juristischen Rahmen sorgen - Stichwörter sind PSI Richtlinie, GDPR, IPR Regelungen, Nutzungslizenzen - wie auch für das vielfältige Angebot an Diensten, die über EOSC-Exchange angeboten werden sollen. Bereits jetzt sind zusätzlich zu den 27 Mitgliedsstaaten weitere zehn Associated Countries und das über Brexit ausgeschiedene Vereinigte Königreich an einer Teilnahme an EOSC interessiert und involviert. Sämtliche bedeutende Forschungsförderer - über Science Europe vertreten - nehmen an den Bemühungen zum Aufbau der EOSC teil. Es wird eine Erfolgstory werden.

\section{Perspektive der Bibliotheken}

Bauer: Du arbeitest seit 1991 Jahren im universitären Umfeld und hast in dieser Zeit durchwegs innovative Projekte - Repositorien bzw. Forschungsdatenmanagement - betrieben. Dabei handelte es sich jeweils um Bereiche, die mittlerweile wie selbstverständlich zum Aufgabengebiet von Bibliotheken gehören. Wie sollen sich Deiner Meinung nach wissenschaftliche Bibliotheken positionieren, um ihre jahrhundertelange Rolle als unterstützende Einrichtungen für Forschung und Lehre auch in Zukunft zeitgemäß erfüllen zu können? Welche Empfehlungen würdest Du Bibliotheksleiterinnen und -leitern geben, damit ihre Einrichtungen zukunftsfit werden bzw. bleiben? Und welchen Ratschlag würdest Du jungen Berufseinsteigerinnen und -einsteigern geben, deren berufliches Ziel (um nicht Berufung zu sagen) es ist, in einer Bibliothek tätig zu sein? 
Budroni: Bibliotheken, ihre Mitarbeiterinnen und Mitarbeiter, sowie deren Leitungen mögen weiterhin open minded sein, wach und aufmerksam. Und vor allem selbstbewusst. Die Botschaft, die von den Bibliotheken ausgehen soll, muss klar und deutlich sein: Wir sind es, die das Wissen schon VOR der Erfindung moderner Universitäten aufbereitet, strukturiert und angeboten haben. So möge es weiterhin sein. Das bedeutet nicht, dass alles so weiter betrieben werden soll wie bis jetzt. Evolution und Innovation werden uns dabei begleiten.

Die aktuellen Entwicklungen sind gekennzeichnet vom Konzept des Open Science. Dabei handelt es sich aber um kein neues Phänomen. Wer die großartigen Reste der Bibliothek zu Pergamon kennt, ist sich der Wirkung von Bibliotheken im Altertum bewusst. Medizin, Pharmazie, Philosophie, Jus, Geographie, Geschichte, Rhetorik, die Schriften der Antike, ihre Erkenntnisse wurden ebendort nicht nur tradiert, sondern weiter aufbereitet und zur Verfügung gestellt. Die Basis für die Entstehung unserer modernen Universitäten wurde in den Bibliotheken gelegt, egal ob diese säkular oder religiös ausgerichtet waren, es waren Bibliothekare, die das Wissen zugänglich machten. Um es mit modernen Begriffen auszudrücken: Bibliothekare haben Wissen aufbewahrt, auffındbar, interoperabel - über Glaubensgrenzen, sprachliche Barrieren, über Meere und Kontinente hinweg, zugänglich und wiederverwendbar gemacht. Es waren Buchdrucker und Bibliothekare, die im 15. Jahrhundert, im damaligen Silicon Valley rund um die Stadt Mainz/Mayence/Magonza jene Techniken perfektionierten, die es möglich machten, dass das moderne Buch in nur 40 Jahren ganz Europa überflutete. ${ }^{18}$ Und es waren wirtschaftstreibende Buchdrucker und Bibliothekare gemeinsam, die die Republik Venedig in eine Art „Amazon des 15. Jahrhunderts" verwandelten. Ist es nicht verwunderlich, dass Amazon, als eine der mächtigsten wirtschaftstreibenden Entitäten der Welt, ein Konzern ist, der rund um das Buch aufgebaut wurde?

Buchdruck und Alphabetisierungsgrade sind wie Zwillinge in unserer Kulturgeschichte groß geworden. Nun sind wir dabei, die typographische Welt zu verlassen und uns mit vollen Zügen der digitalen Welt zuzuwenden. Das geschieht in Europa. Wir sind dabei, nicht nur fußfrei erste Reihe, sondern gleich auf der Hauptbühne.

Die Bibliotheken zeigen auf, wie man moderne Managementmethoden anwendet, um hochkomplexe Projekte für unsere Universitäten zu akquirieren. „Nur zu!“", das möchte ich jenen jungen Kolleginnen und Kollegen mitteilen. Traut euch Daten zu modellieren, disziplinenübergreifend zu denken und zu handeln. Ihr könnt wunderbare Mediziner, Juristen, Philologen, Physiker, usw. sein - noch bessere werdet ihr sein, wenn Euch das fundierte Wissen unserer Bibliothekswelt begleitet. 


\section{Paolo Budroni}

Seit September 2019 von der Uni Wien karenziert und an der TU Wien (TU Bibliothek, Internationale Projekte) tätig.

Paolo Budroni leitete 1991 zunächst das Büro für Wissenschaftsdokumentation am Außeninstitut, wo er mit dem Aufbau der ersten Forschungsdokumentation der Universität Wien (DonKey) betraut wurde. 19951997 Mitherausgeber der Zeitschrift Die Universiät/TheUniversity/LaUniversidad. 1997-98

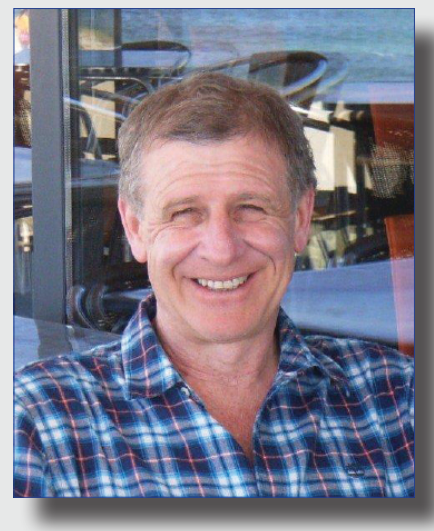
koordinierte er am Logistischen Zentrum der Universität das Teilprojekt „Forschungsstrategien an der Universität Wien“. Seit 2005 an der Universitätsbibliothek Wien und seit 2007 als Leiter des Projekts für digitale Langzeitarchivierung Phaidra tätig, das von ihm und Thomas Wana (ZID) konzipiert und entworfen wurde. Phaidra wird derzeit in fünf Länder und an 17 Institutionen verwendet.

1986 Dr. phil. mit einer semiotischen Untersuchung über Don Camillo und Peppone. 1988 Abschluss eines Lehrganges an der Wirtschaftsuniversität Wien und 1996 Abschluss der Europäischen Akademie des Bundes mit einem Postgraduate Degree in European Integration for Public Administration. Während einer mehrjährigen Karenzzeit war er als Dozent für Marketing (Master-Kurse) an der Università degli Studi di Perugia (Scienze della Comunicazione) sowie als Marketingleiter und Strategic Business Development Manager für ein deutsches Telekommunikationsunternehmen tätig.

Europäische Projekte (geleitet oder akquiriert, oder an der Akquise beteiligt): OpenAIRE, TEMPUS im Westbalkanraum, Europeana Libraries, LEARN, EOSC Secretariat, EOSC Pillar, CO-Versatile (Covid19 Urgent Action).

\section{Aktuelle Mitgliedschaften:}

- Austrian National Delegate in E-Infrastructure Reflection Group (E-IRG) and since 2020 Chair of the E-IRG

- Member of board of the Austrian RDA National Node

- Member of the H2020 Project EOSC Secretariat, serving in Sub Task Researchers Engagement

- Counselling Partner of University of Vienna, H2020 Project EOSC Pillar 
- Coordinator of Task Force KPIs (EOSC WG Landscape)

- Nominated expert in EOSC WG Landscape

- Nominated expert in EOSC WG Sustainability

- Member of the SRIA EOSC Editorial Board

- Member of the EOSC WG Bylaws

\section{Publikationen (in Auswahl):}

Paolo Budroni, Jean-Claude Burgelman, Michel Schouppe, Architectures of Knowledge (2019): The European Open Science Cloud, ABI Technik 39(2), 130-141. http://doi.org./10.1515/abitech-2019-2016

Paolo Budroni (2017): Survey on open research data. Working Group BISA 2017 - Riflessioni sugli esiti delle iniziative BISA e progetto eInfrastructures Austria. In: Results of BISA (Bibliosan per la Scienza Aperta) - Rapporti ISTISAN 17/32, 28-39. http://old.iss.it/binary/ publ/cont/17_32_web.pdf

Paolo Budroni et al. (2017): RDA Europe Workshop - From Planning to Action. Towards the Establishment of an Austrian Research Infrastructure (Wien, 23. November 2017). Mitteilungen der VÖB 70(34), 382-389. https://doi.org/10.31263/voebm.v70i3.1962

Paolo Budroni et al. (2017): Development of a Model Policy for Research Data Management (RDM) at Austrian Research Institutions. A Use Case. In: LEARN (Ed.), LEARN Toolkit of Best Practice for Research Data Management. London: Leaders Activating Research Networks (LEARN), 12-16. https://doi.org/10.14324/000.learn.03 Paolo Budroni et al. (2017): Model Policy for Research Data Management (RDM) at Research Institutions/Institutes. In: LEARN (Ed.), LEARN Toolkit of Best Practice for Research Data Management. London: Leaders Activating Research Networks (LEARN), 133-136. https://doi.org/10.14324/000.learn.26

Paolo Budroni et al. (2017): Guidance for Developing a Research Data Management (RDM) Policy. In: LEARN (Ed.), LEARN Toolkit of Best Practice for Research Data Management. London: Leaders Activating Research Networks (LEARN), 137-140. https://doi. org/10.14324/000.learn.27

Paolo Budroni et al. (2017): Evaluation Grid of RDM Policies in Europe. In: LEARN (Ed.), LEARN Toolkit of Best Practice for Research Data Management. London: Leaders Activating Research Networks (LEARN), 141-168. https://doi.org/10.14324/000.learn.28 
Raman Ganguly, Paolo Budroni, Barbara Sánchez Solís (2017): Living Digital Ecosystems for Data Preservation - An Austrian Use Case Towards the European Open Science Cloud. In: L. Chan and F. Loizides (Eds.), Expanding Perspectives on Open Science: Communities, Cultures and Diversity in Concepts and Practices, Amsterdam: IOS Press, 203-210. https://doi.org/10.3233/978-1-61499-769-6-203

Tomasz Miksa, Andreas Rauber, Raman Ganguly, Paolo Budroni (2017): Information Integration for Machine Actionable Data Management Plans. International Journal of Digital Curation 12(1), 22-35. https://doi.org/10.2218/ijdc.v12i1.529

Paolo Budroni (2016): Good Governance - Strukturen zur Sicherung der Nachhaltigkeit von Projekten im akademischen Umfeld. Das nationale Projekt e-Infrastructures Austria. ABI Technik 36(1), 24-36. https://doi.org/10.1515/abitech-2016-0002

Mag. Bruno Bauer

ORCID: http://orcid.org/0000-0002-4729-331X Medizinische Universität Wien, Universitätsbibliothek

E-Mail: bruno.bauer@meduniwien.ac.at

Dr. Paolo Budroni

ORCID: https://orcid.org/0000-0001-7490-5716

TU Wien, Bibliothek

E-Mail: paolo.budroni@tuwien.ac.at 
1 Paolo Budroni (1997): Über Forschung und Entwicklung (F\&E) Technologietransfer und F\&E Politik als Strategische Instrumente der Wirtschaftspolitik“. AP Nr. 1, Verwaltungsakademie des Bundes, Europaakademie: Wien.

2 EUROVOC, Amt für Öffentliche Veröffentlichungen der Europäischen Gemeinschaften, Luxemburg 1995.

3 http://learn-rdm.eu/en/about/

4 LERU Roadmap for Research Data (LERU Research Data Working Group), Advice Paper No. 14 - December 2013. https://www.leru.org/ files/LERU-Roadmap-for-Research-Data-Full-paper.pdf

5 Results of BISA (Bibliosan per la Scienza Aperta) - Rapporti ISTISAN 17/32. http://old.iss.it/binary/publ/cont/17_32_web.pdf

6 https://nadreweb.ethernet.edu.et/ und http://webcms.uct.ac.za/sites/ default/files/image_tool/images/509/Presentations/Day_two/Transforming\%20Research\%20 Landscape\%20 in\%20Africa\%20using\%20 Open\%20Access\%2C\%20the\%20case\%20of\%20Ethiopia\%20-\%205\%20 December\%202019.pdf

7 https://ec.europa.eu/neighbourhood-enlargement/tenders/taiex_en

8 http://e-irg.eu/about

9 http://e-irg.eu/executiveboard

10 https://eosc-launch.eu/declaration/

11 Paolo Budroni, Jean-Claude Burgelman, Michel Schouppe, Architectures of Knowledge (2019): The European Open Science Cloud, ABI Technik 39(2), 130-141. http://doi.org./10.1515/abitech-2019-2016

12 Paolo Budroni, Stefan Hanslik, Barbara Sánchez Solís: Developing EOSC - A view on an ongoing process, as per 31 January 2020. Online unter: https://www.aco.net/developing_eosc.html?L=1

13 https://www.eoscsecretariat.eu/working-groups/landscape-working-group

14 https://www.eoscsecretariat.eu/working-groups/sustainability-working-group

15 https://www.eoscsecretariat.eu/eosc-governance/eosc-executive-board

16 https://www.eoscsecretariat.eu/eosc-governance/eosc-governance-board

17 Statuten der EOSC Association. https://www.eoscsecretariat.eu/sites/ default/files/eosc_statutes.pdf

18 Cristina Dondi (ed.) (2018): Printing revolution 1450-1500. I cinquant'anni che hanno cambiato l'Europa. Catalogo della mostra (Venezia, 1 settembre 2018-7 gennaio 2019). Ediz. italiana e inglese. 